\title{
Bio Efficacy of Fungicides and Bio Agents against Colletotrichum gloeosporioides causing Anthracnose in Pomegranate
}

\author{
Vandana, R. K. Mesta, K. C. Kiran Kumar, M. D. Jameel Jhalegar and Siddanna Thoke \\ Department of plant pathology, College of Horticulture, Bagalkot, Karnataka, India \\ *Corresponding author
}

\section{A B S T R A C T}

\section{Keywords}

Pomegranate, anthracnose, Colletotrichum gloeosporioides, bio agents, fungicides, per cent disease index

Article Info

Accepted:

20 June 2021

Available Online:

10 July 2021
Pomegranate (Punica granatum L.) of Lythraceae family, called "fruit of paradise" is one of the major fruit crops of arid region. At present, the known fungal disease agents of pomegranate are Colletotrichum gloeosporioides, Alternaria spp., Botrytis cinerea, Aspergillus niger, Pestalotiopsis versicolor, Penicillium spp. and Rhizopus spp. Among the various fungal diseases, anthracnose caused by Colletotrichum gloeosporioides is the most important post harvest diseases in pomegranate. In this study, several fungicides and bio agents were evaluated against this pathogen. Among systemic fungicides, propiconazole $25 \% \mathrm{EC}$, hexaconazole $5 \% \mathrm{EC}$, iprobenfos $48 \%$ EC and kresoxim methyl $44.3 \%$ EC recorded cent per cent mycelial inhibition of Colletotrichum gloeosporioides. Among six contact and seven combi products (1000, 1500, 2000 and $3000 \mathrm{ppm}$ ), mancozeb 75\% WP, zineb 75\% WP, tricyclazole $18 \%+$ mancozeb $62 \%$, carbendazim $12 \%+$ mancozeb $63 \%$ and carboxin $37.5 \%+$ thiram $37.5 \%$ completely inhibited the growth of Colletotrichum gloeosporioide. Among different bio agents tested, Trichoderma harzianum, UAS, Dharwad isolate recorded significantly higher per cent mean inhibition followed by Trichoderma harzianum, UHS, Bagalkot isolate. Fungicides and bio agents found effective were tested in field condition for the management of pomegranate leaf spot and fruit rot. The results showed that mancozeb 75\% WP, carbendazim 12\% + mancozeb $63 \%$ and propiconazole 25\% EC @ 0.1\% were effective in controlling the leaf spot and fruit rot incidence as compared to other treatments.

\section{Introduction}

Pomegranate (Punica granatum L.) is an ancient fruit of arid regions of the world. It belongs to the family Lythraceae. Pomegranate is native from Iran to Himalayas in northern India. It is mainly liked for its refreshing juice. Pomegranates are also used in cooking, juice blends, meal garnishes and alcoholic beverages. It is having potential to counteract free radicals and provide antioxidants, tannins and anthocyanins. It has been used to treat sore throats, coughs, skin disorders and digestive disorders. (Bhowmik et al., 2013). India is world's largest producer of the pomegranate fruit followed by Iran, 
China, Turkey and United States. Maharashtra is the leading producer of pomegranate followed by Karnataka, Gujarat, Madhya Pradesh, Himachal Pradesh and Rajasthan. Area under pomegranate cultivation in India is 2, 34,000 ha with the production of 28, 45,000 MT during 2017-18. Karnataka accounts 25,970 ha area, 2, 68,230 MT production and 10.33 MT/ha productivity during 2017-18. It has spread across different districts of Karnataka viz., Chitradurga, Bellary, Tumkur, Vijayapur and Bagalkot (Anon., 2018).

Pomegranate is affected by several pathogens before and after harvest. This results in huge losses during harvest and storage. Fruit rot is one of the contributing factors for the low productivity. Fruit rot is wide spread in rainy season/ high moisture conditions. It is suspected to be caused by, Colletotrichum sp., Alternaria sp., Cercospora sp., Phytophthora sp., Penicillium spp., Aspergillus spp., Coniella granati and Rhizopus spp. (Munhuweyia et al., 2016). Among these pathogens, Colletotrichm gloeosporioides (Penz.) Penz. \& Sacc. causing anthracnose important fruit rot (pre and post harvest) pathogen in pomegranate. In India, Colletotrichm gloeosporioides responsible for leaf spot disease was first time reported on coffee by Butler (1918) and anthracnose disease on mango by McRae (1924).

In India, Chandra and Tondon (1965) for the first time reported $C$. gloeosporioides causing pomegranate leaf spot and fruit rot. The pre harvest management of anthracnose is necessary as the pathogen is known to cause huge loss after harvest. But there are several fungicides available in the market and their bio efficacy need to evaluate under in vitro and field studies. Therefore, the efficacy of different contact, systemic and combination fungicides and bio agents were verified by in vitro and effective fungicides and bio agents were tested under field condition.

\section{Materials and Methods}

The experimental studies on pomegranate anthracnose were carried out at Department of Plant Pathology, College of Horticulture, Bagalkot. Pre harvest management study was carried out at farmer field at Tulasigeri village of Bagalkote taluk. The standard tissue isolation technique was followed for the isolation of the pathogens causing anthracnose. Pure culture was done by following single spore isolation technique. Isolated pathogen was identified through the cultural, morphological and molecular studies.

\section{In vitro evaluation of fungicides and bio agents against Colletotrichum gloeosporioides}

Twenty four fungicides evaluated against the Colletotrichum gloeosporioides to test their efficacy under the laboratory condition. The systemic fungicides were evaluated at the concentration of 250, 500, 1000 and 1500 ppm. The contact and combi products were evaluated at concentration of 1000, 2000, 2500 and $3000 \mathrm{ppm}$. The fungicides were evaluated using potato dextrose agar as the basal medium and by following Poison Food Technique. Antagonistic micro organisms like, Bacillus subtilis, Trichoderma asperellum and Trichoderma harzianum were evaluated for their antagonistic properties by dual plate culture technique.

Field evaluation of fungicides and bio control agents against fruit rot of pomegranate

The experiments on field evaluation of fungicides and bio control agents against fruit rot of pomegranate was conducted during July 2019 in natural epiphytic conditions on pomegranate at farmer field in Tulasigeri village of Bagalkote taluk. The fungicides and commercial formulation of biological agents 
which were found effective in vitro were tested at recommended concentration. There were ten treatments for fruit rot disease with randomized completely blocked design (RCBD) and each treatment was replicated three times. Two sprays were taken at 40 and 60 days after fruit set. The treatment details were as follows.

\section{Results and Discussion}

In vitro evaluation of contact fungicides against Colletotrichum gloeosporioides (Fig.1 and Table.1)

Mancozeb 75\% WP and zineb 75\% WP showed cent per cent inhibition of mean mycelial growth and were found to be most effective as compared to other four contact fungicides. The least mean per cent inhibition was recorded by copper oxy chloride 50\% WP $(33.33 \%)$ and propineb $70 \%$ WP $(49.62 \%)$.

At 1000 ppm, mancozeb 75\% WP and zineb $75 \% \mathrm{WP}$ were found to be most effective with complete inhibition of mycelia growth followed by captan 50\% WP (59.26\%). Copper oxy chloride 50\% WP was least effective $(2.59 \%)$. The same trend continued at 2000 and $2500 \mathrm{ppm}$ concentrations as all fungicides were slightly effective than lower concentrations. At 3000 ppm, mancozeb 75\% $\mathrm{WP}$, zineb $75 \% \mathrm{WP}$, propineb $70 \% \mathrm{WP}$ and chlorothalonil $75 \%$ WP recorded complete (100.00\%) fungal growth inhibition. Copper oxy chloride 50\% WP showed least per cent inhibition of mycelia growth $(47.41 \%)$.

In vitro evaluation of systemic fungicides against Colletotrichum gloeosporioides (Fig.2 and Table.2)

Total eleven systemic fungicides were evaluated at four concentrations for their efficacy against Colletotrichum gloeosporioides. Four systemic fungicides viz., hexaconazole 5\% EC, propiconazole $25 \%$ EC, kresoxim methyl $44.3 \%$ EC and iprobenfos $48 \%$ EC showed cent per cent inhibition of mean mycelial growth and were found to be most effective as compared to other seven systemic fungicides. The next best chemicals were found to be tebuconazole $25 \%$ EC, difenconazole $25 \% \mathrm{EC}$ and tricyclazole $75 \%$ WP with $97.47 \%, 89.75 \%$ and $89.02 \%$ mean per cent inhibition of mycelia growth. The least growth inhibition was recorded by thifluzamide $24 \%$ SC $(9.93 \%)$ followed by azoxystrobin $25 \%$ EC (22.97\%).

At 250 ppm, cent per cent inhibition of mycelia growth was recorded by hexaconazole $5 \%$ EC, propiconazole $25 \%$ EC, kresoxim methyl $44.3 \%$ EC and iprobenfos $48 \%$ EC and were found best chemicals followed by tebuconazole $25 \% \quad \mathrm{EC} \quad(93.45 \%)$, difenconazole $25 \%$ EC $(84.44 \%)$ and tricyclazole $75 \%$ WP $(71.63 \%)$. Thifluzamide $24 \%$ SC and azoxystrobin $25 \%$ EC did not show inhibition at this concentration level. At $500 \mathrm{ppm}$ the same trend continued. In addition to these 4 effective fungicides, showed cent per cent inhibition was recorded by tricyclazole $75 \% \mathrm{WP}$ at 1000 and $1500 \mathrm{ppm}$, and by difencinazole at 1500 ppm. Carbendazim 50\% WP counld show inhibition of $88.63 \%$ at $1500 \mathrm{ppm}$. All other fungicides were ineffective.

\section{In vitro evaluation of combi products against Colletotrichum gloeosporioides} (Fig.3 and Table.3)

Total seven combi products were evaluated for their efficacy against Colletotrichum gloeosporioides at 1000, 2000, 2500 and 3000 ppm.

Mean per cent inhibition of mycelia growth were recorded for all the different combi products, among which tricyclazole $18 \%+$ mancozeb 62\%, carbendazim $12 \%+$ 
mancozeb $63 \%$ and carboxin $37.5 \%+$ thiram $37.5 \%$ showed complete inhibition. The efficacy of captan $70 \%+$ hexaconazole $5 \%$ $(79.07 \%)$ was much the same as tricyclazole $45 \%+$ hexaconazole $10 \% \quad(77.40 \%)$. Hexaconazole $4 \%+$ zineb $68 \%$ were least effective, which recorded $70.45 \%$ mycelia growth inhibition.

At 1000 ppm, cent per cent inhibition was recorded by tricyclazole $18 \%+$ mancozeb $62 \%$, carbendazim $12 \%+$ mancozeb $63 \%$ and carboxin $37.5 \%+$ thiram $37.5 \%$ and these were followed by captan $70 \%+$ hexaconazole $5 \%(74.81 \%)$. Least efficacy was observed for hexaconazole $4 \%+$ zineb $68 \%$ (59.25\%). The same trend continued even atv2000 and 2500 ppm concentrations At 3000 ppm, cent per cent inhibition was recorded by tricyclazole $18 \%+$ mancozeb $62 \%$, carbendazim $12 \%+$ mancozeb $63 \%$ and carboxin $37.5 \%+$ thiram $37.5 \%$. Tricyclazole $45 \%+$ hexaconazole $10 \%$ and hexaconazole $4 \%+$ zineb $68 \%$ were recorded next highest value of per cent mycelial inhibition (88.88\%).

In vitro evaluation of bio agents against Colletotrichum gloeosporioides (Fig.4 and Table.4)

Four different bio agents collected from UAS, Dharwad and UHS, Bagalkot were tested for their competitive ability against Colletotrichum gloeosporioides. Trichoderma harzianum, UAS, Dharwad isolate recorded significantly higher per cent mean inhibition (86.67\%) followed by Trichoderma harzianum, UHA, Bagalkot isolate (75.19\%). Trichoderma asperellum, UHS, Bagalkot isolate showed $69.63 \%$ growth inhibition. The per cent inhibition of Colletotrichum gloeosporioides by bacterial antagonists, Pseudomonas fluorescens (58.15\%) and Bacillus subtilis (56.67\%) isolates of UAS, Dharwad and Pseudomonas fluorescens, UHS, Bagalkot isolate $(55.93 \%)$ was on par with each other. The least per cent inhibition was recorded for UHS, Bagalkot isolate of Bacillus subtilis $(54.44 \%)$.

Field management of leaf spot and fruit rot of pomegranate (Fig5, Table5 and Table6)

20 days after second spray, highest per cent disease reduction over control was recorded in carbendazim $12 \%$ + mancozeb $63 \%(56.52 \%)$ @ $0.1 \%$, mancozeb 75\% WP @ $0.1 \%$ $(54.35 \%)$ and propiconazole 25\% EC @ $0.025 \%(53.26 \%)$ with lowest leaf spot PDI values of $26.67 \%, 28.00 \%$ and $28.67 \%$ respectively. Significant PDI of fruit rot was recorded for propiconazole 25\% EC @ $0.025 \%(36.00 \%)$, mancozeb 75\% WP @ $0.1 \%(37.33 \%)$ and carbendazim 12\%+ mancozeb 63\%@0.1\% (37.33\%) followed by iprobenfos 48\% EC @ 0.025\% (42.67\%) and kresoxim methyl 44.3\% EC @ $0.025 \%$ (42.67\%). Highest PDI was recorded in control (57.33\%), T. harzianum @1\% $(49.33 \%)$ and tricyclazole $18 \%+$ mancozeb 62\%@0.1\% (48.00\%). These results showed that Mancozeb 75\% WP, carbendazim 12\%+ mancozeb $63 \%$ and propiconazole $25 \%$ EC were effective in controlling the leaf spot and fruit rot incidence as compared to other treatments. Among systemic fungicides (250, 500, 1000 and $1500 \mathrm{ppm}$ ), propiconazole $25 \%$ $\mathrm{EC}$, hexaconazole $5 \% \mathrm{EC}$, iprobenfos $48 \% \mathrm{EC}$ and kresoxim methyl $44.3 \%$ EC recorded cent per cent mycelial inhibition at their lowest level of test concentration. Least per cent inhibition was recored by thifluzamide $24 \%$ SC $(9.93 \%)$ followed by azoxystrobin $25 \%$ EC (22.97\%). Among six contact and seven combi products (1000, 1500, 2000 and 3000 ppm), mancozeb $75 \% \mathrm{WP}$, zineb $75 \% \mathrm{WP}$, tricyclazole $18 \%+$ mancozeb $62 \%$, carbendazim $12 \%+$ mancozeb $63 \%$ and carboxin $37.5 \%$ + thiram $37.5 \%$ completely inhibited the growth of both the test pathogens at their lowest level of concentrations evaluated. 
Table.1 In vitro efficacy of contact fungicides against Colletotrichum gloeosporioides

\begin{tabular}{|c|c|c|c|c|c|c|}
\hline \multirow[t]{3}{*}{ Sl. No. } & \multirow[t]{3}{*}{ Fungicide } & \multicolumn{5}{|c|}{ Per cent inhibition of mycelial growth } \\
\hline & & \multicolumn{5}{|c|}{ Concentration (ppm) } \\
\hline & & 1000 & 2000 & 2500 & 3000 & Mean \\
\hline 1 & Captan $75 \%$ WP & $\begin{array}{c}59.26 \\
*(50.33)\end{array}$ & $\begin{array}{c}62.59 \\
(52.29)\end{array}$ & $\begin{array}{c}64.44 \\
(53.39)\end{array}$ & $\begin{array}{c}67.41 \\
(55.19)\end{array}$ & 63.42 \\
\hline 2 & Chlorothalonil 75\% WP & $\begin{array}{c}12.96 \\
(21.09)\end{array}$ & $\begin{array}{c}38.15 \\
(38.14)\end{array}$ & $\begin{array}{c}78.15 \\
(62.13)\end{array}$ & $\begin{array}{l}100.00 \\
(89.66)\end{array}$ & $\mathbf{5 7 . 3 1}$ \\
\hline 3 & Copper oxychloride $50 \%$ WP & $\begin{array}{c}2.59 \\
(9.21)\end{array}$ & $\begin{array}{c}38.15 \\
(38.14)\end{array}$ & $\begin{array}{c}45.19 \\
(42.23)\end{array}$ & $\begin{array}{c}47.41 \\
(43.51)\end{array}$ & 33.33 \\
\hline 4 & Mancozeb $75 \%$ WP & $\begin{array}{r}100.00 \\
(89.75)\end{array}$ & $\begin{array}{l}100.00 \\
(89.75)\end{array}$ & $\begin{array}{r}100.00 \\
(89.75)\end{array}$ & $\begin{array}{l}100.00 \\
(89.75)\end{array}$ & 100.00 \\
\hline 5 & Propineb 50\% WP & $\begin{array}{c}23.33 \\
(28.87)\end{array}$ & $\begin{array}{c}26.67 \\
(31.08)\end{array}$ & $\begin{array}{c}48.52 \\
(44.15)\end{array}$ & $\begin{array}{l}100.00 \\
(89.66)\end{array}$ & 49.62 \\
\hline 6 & Zineb $75 \% \mathrm{WP}$ & $\begin{array}{r}100.00 \\
(89.75)\end{array}$ & $\begin{array}{l}100.00 \\
(89.75)\end{array}$ & $\begin{array}{r}100.00 \\
(89.75)\end{array}$ & $\begin{array}{l}100.00 \\
(89.75)\end{array}$ & 100.00 \\
\hline \multirow{2}{*}{\multicolumn{2}{|c|}{ Mean }} & 49.68 & 60.92 & 72.71 & 85.80 & \\
\hline & & $\mathbf{F}$ & $\mathbf{C}$ & \multicolumn{3}{|c|}{$\mathbf{F} \times \mathbf{C}$} \\
\hline & S.Em \pm & 0.19 & 0.16 & \multicolumn{3}{|c|}{0.39} \\
\hline & CD@ 0.01 & 0.72 & 0.59 & \multicolumn{3}{|c|}{1.44} \\
\hline
\end{tabular}

*Arcsine Transformed Values 
Table.2 In vitro efficacy of systemic fungicides against Colletotrichum gloeosporioides

\begin{tabular}{|c|c|c|c|c|c|c|}
\hline \multirow{3}{*}{$\begin{array}{c}\text { Sl. } \\
\text { No. }\end{array}$} & \multirow[t]{3}{*}{ Fungicide } & \multicolumn{5}{|c|}{ Per cent inhibition of mycelial growth } \\
\hline & & \multicolumn{5}{|c|}{ Concentration (ppm) } \\
\hline & & 250 & 500 & 1000 & 1500 & Mean \\
\hline 1 & Azoxystrobin $25 \%$ EC & $\begin{array}{c}0.00 \\
*(0.249)\end{array}$ & $\begin{array}{c}7.43 \\
(15.82)\end{array}$ & $\begin{array}{c}33.22 \\
(35.19)\end{array}$ & $\begin{array}{c}51.26 \\
(45.72)\end{array}$ & 22.97 \\
\hline 2 & Carbendazim $50 \%$ EC & $\begin{array}{c}62.67 \\
(52.33)\end{array}$ & $\begin{array}{c}71.33 \\
(57.63)\end{array}$ & $\begin{array}{c}72.41 \\
(58.31)\end{array}$ & $\begin{array}{c}88.63 \\
(70.31)\end{array}$ & 73.76 \\
\hline 3 & Difenconazole $25 \% \mathrm{EC}$ & $\begin{array}{c}84.44 \\
(66.77)\end{array}$ & $\begin{array}{c}86.71 \\
(68.61)\end{array}$ & $\begin{array}{c}87.88 \\
(69.66)\end{array}$ & $\begin{array}{r}100.00 \\
(89.75)\end{array}$ & 89.75 \\
\hline 4 & Hexaconazole $5 \%$ EC & $\begin{array}{l}100.00 \\
(89.75)\end{array}$ & $\begin{array}{l}100.00 \\
(89.75)\end{array}$ & $\begin{array}{l}100.00 \\
(89.75)\end{array}$ & $\begin{array}{l}100.00 \\
(89.75)\end{array}$ & 100.00 \\
\hline 5 & Iprobenfos $48 \% \mathrm{EC}$ & $\begin{array}{l}100.00 \\
(89.75)\end{array}$ & $\begin{array}{l}100.00 \\
(89.75)\end{array}$ & $\begin{array}{l}100.00 \\
(89.75)\end{array}$ & $\begin{array}{l}100.00 \\
(89.75)\end{array}$ & 100.00 \\
\hline 6 & Kresoxim methyl $44.3 \% \mathrm{EC}$ & $\begin{array}{l}100.00 \\
(89.75)\end{array}$ & $\begin{array}{r}100.00 \\
(89.75)\end{array}$ & $\begin{array}{l}100.00 \\
(89.75)\end{array}$ & $\begin{array}{l}100.00 \\
(89.75)\end{array}$ & 100.00 \\
\hline 7 & Propiconazole $25 \%$ EC & $\begin{array}{l}100.00 \\
(89.75)\end{array}$ & $\begin{array}{l}100.00 \\
(89.75)\end{array}$ & $\begin{array}{l}100.00 \\
(89.75)\end{array}$ & $\begin{array}{l}100.00 \\
(89.75)\end{array}$ & 100.00 \\
\hline 8 & Tebuconazole $25 \%$ EC & $\begin{array}{c}93.45 \\
(75.21)\end{array}$ & $\begin{array}{c}96.45 \\
(79.33)\end{array}$ & $\begin{array}{l}100.00 \\
(89.75)\end{array}$ & $\begin{array}{l}100.00 \\
(89.75)\end{array}$ & 97.47 \\
\hline 9 & Thifluzamide $24 \%$ EC & $\begin{array}{c}0.00 \\
(0.24)\end{array}$ & $\begin{array}{c}4.61 \\
(12.37)\end{array}$ & $\begin{array}{c}12.11 \\
(20.35)\end{array}$ & $\begin{array}{c}23.03 \\
(28.64)\end{array}$ & 9.93 \\
\hline 10 & Thiophonate methyl 70\% WP & $\begin{array}{c}44.45 \\
(41.81)\end{array}$ & $\begin{array}{c}45.19 \\
(42.24)\end{array}$ & $\begin{array}{c}47.74 \\
(43.66)\end{array}$ & $\begin{array}{c}52.93 \\
(46.67)\end{array}$ & 47.56 \\
\hline 11 & Tricyclazole $75 \% \mathrm{WP}$ & $\begin{array}{c}71.63 \\
(57.82)\end{array}$ & $\begin{array}{c}84.48 \\
(66.81)\end{array}$ & $\begin{array}{l}100.00 \\
(89.75)\end{array}$ & $\begin{array}{l}100.00 \\
(89.75)\end{array}$ & 89.02 \\
\hline \multirow{2}{*}{\multicolumn{2}{|c|}{ Mean }} & 68.78 & 72.38 & $\mathbf{7 7 . 5 7}$ & 83.26 & \\
\hline & & $\mathbf{F}$ & $\mathbf{C}$ & \multicolumn{3}{|c|}{$\mathbf{F} \times \mathbf{C}$} \\
\hline & S.Em \pm & 0.26 & 0.16 & \multicolumn{3}{|c|}{0.51} \\
\hline & CD @ 0.01 & 0.97 & 0.58 & \multicolumn{3}{|c|}{1.93} \\
\hline
\end{tabular}

*Arcsine Transformed Values 
Table.3 In vitro efficacy of combi products against Colletotrichum gloeosporioides

\begin{tabular}{|c|c|c|c|c|c|c|}
\hline \multirow[t]{3}{*}{ Sl. No. } & \multirow[t]{3}{*}{ Fungicide } & \multirow{2}{*}{\multicolumn{5}{|c|}{$\begin{array}{c}\text { Per cent inhibition of mycelial growth } \\
\text { Concentration (ppm) }\end{array}$}} \\
\hline & & & & & & \\
\hline & & 1000 & 2000 & 2500 & 3000 & Mean \\
\hline 1 & Captan $70 \%+$ hexaconazole $5 \%$ & $\begin{array}{c}74.81 \\
*(59.87)\end{array}$ & $\begin{array}{c}77.41 \\
(61.63)\end{array}$ & $\begin{array}{c}79.62 \\
(63.17)\end{array}$ & $\begin{array}{c}84.44 \\
(66.77)\end{array}$ & 79.07 \\
\hline 2 & Carbendazim $12 \%+$ mancozeb $63 \%$ & $\begin{array}{l}100.00 \\
(89.69)\end{array}$ & $\begin{array}{l}100.00 \\
(89.69)\end{array}$ & $\begin{array}{l}100.00 \\
(89.69)\end{array}$ & $\begin{array}{l}100.00 \\
(89.69)\end{array}$ & 100.00 \\
\hline 3 & Carboxin $37.5 \%$ thiram $37.5 \%$ & $\begin{array}{l}100.00 \\
(89.69)\end{array}$ & $\begin{array}{l}100.00 \\
(89.69)\end{array}$ & $\begin{array}{l}100.00 \\
(89.69)\end{array}$ & $\begin{array}{l}100.00 \\
(89.69)\end{array}$ & 100.00 \\
\hline 4 & Hexaconazole $4 \%+$ zineb $68 \%$ & $\begin{array}{c}59.25 \\
(50.33)\end{array}$ & $\begin{array}{c}61.85 \\
(51.85)\end{array}$ & $\begin{array}{c}71.85 \\
(57.96)\end{array}$ & $\begin{array}{c}88.88 \\
(70.51)\end{array}$ & 70.45 \\
\hline 5 & Tebuconazole $50 \%+$ trifluoxystrobin $25 \%$ & $\begin{array}{c}69.26 \\
(56.32)\end{array}$ & $\begin{array}{c}71.48 \\
(57.72)\end{array}$ & $\begin{array}{c}75.55 \\
(60.37)\end{array}$ & $\begin{array}{c}78.51 \\
(62.39)\end{array}$ & 73.70 \\
\hline 6 & Tricyclazole $18 \%+$ mancozeb $62 \%$ & $\begin{array}{l}100.00 \\
(89.69)\end{array}$ & $\begin{array}{l}100.00 \\
(89.69)\end{array}$ & $\begin{array}{l}100.00 \\
(89.69)\end{array}$ & $\begin{array}{l}100.00 \\
(89.69)\end{array}$ & 100.00 \\
\hline 7 & Tricyclazole $45 \%$ + hexaconazole $10 \%$ & $\begin{array}{c}63.70 \\
(52.95)\end{array}$ & $\begin{array}{c}71.48 \\
(57.72)\end{array}$ & $\begin{array}{c}85.55 \\
(67.67)\end{array}$ & $\begin{array}{c}88.88 \\
(70.54)\end{array}$ & 77.40 \\
\hline \multirow{2}{*}{\multicolumn{2}{|c|}{ Mean }} & 81.00 & 83.17 & 87.51 & 91.52 & \\
\hline & & $\mathbf{F}$ & C & \multicolumn{3}{|c|}{$\mathbf{F} \times \mathbf{C}$} \\
\hline & S.Em \pm & 0.27 & 0.20 & \multicolumn{3}{|c|}{0.54} \\
\hline & CD@0.01 & 0.99 & 0.75 & \multicolumn{3}{|c|}{1.98} \\
\hline
\end{tabular}

*Arcsine Transformed Values 
Table.4 In vitro efficacy of bio agents against Colletotrichum gloeosporioides

\begin{tabular}{|c|c|c|}
\hline \multirow{2}{*}{ Sl. No. } & Bio agent & Per cent inhibition of mycelial growth \\
\cline { 3 - 3 } & & Colletotrichum gloeosporioides \\
\hline $\mathbf{1}$ & T. harzianum (UHS, Bagalkot) & 75.19 \\
& & $*(60.10)$ \\
\hline $\mathbf{2}$ & T. harzianum (UAS, Dharwad) & 86.67 \\
& & $(68.57)$ \\
\hline $\mathbf{3}$ & T. asperellum (UHS, Bagalkot) & 69.63 \\
& & $(56.53)$ \\
\hline $\mathbf{4}$ & P. fluorescens (UHS, Bagalkot) & 55.93 \\
& & $(48.39)$ \\
\hline $\mathbf{5}$ & P. fluorescens (UAS, Dharwad) & 58.15 \\
& & $(49.67)$ \\
\hline $\mathbf{6}$ & B. subtilis (UHS, Bagalkot) & 54.44 \\
& & $(47.53)$ \\
\hline $\mathbf{7}$ & B. subtilis (UAS, Dharwad) & 56.67 \\
& & $(48.81)$ \\
\hline & S.Em \pm & $\mathbf{0 . 6 7}$ \\
\hline & CD @ 0.01 & $\mathbf{2 . 8 3}$ \\
\hline
\end{tabular}

*Arcsine Transformed Values 
Table.5 Efficacy of fungicides and bio control agent against leaf spot of pomegranate

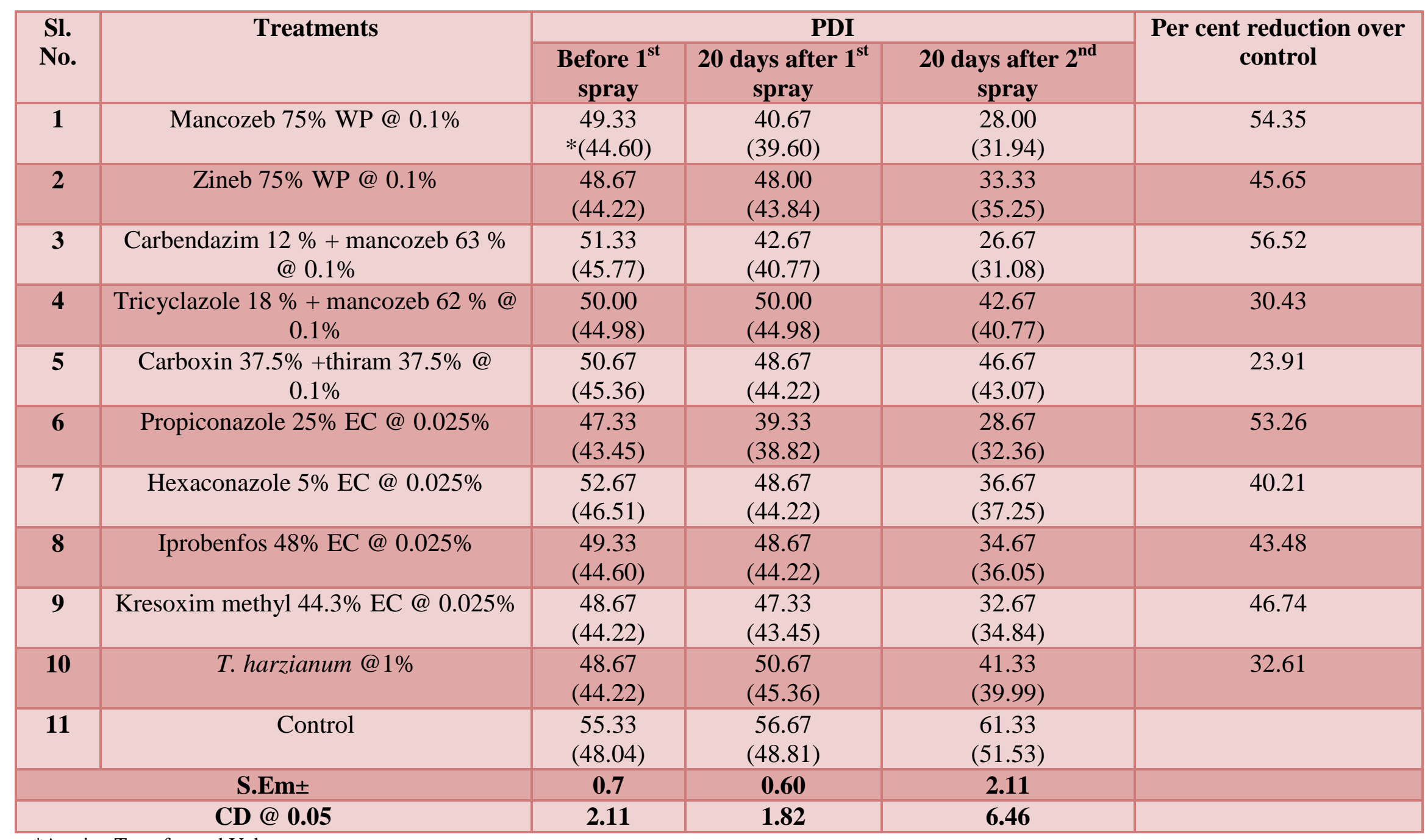

*Arcsine Transformed Values 
Table.6 Efficacy of fungicides and bio control agent against fruit rot of pomegranate

\begin{tabular}{|c|c|c|c|c|c|}
\hline \multirow{2}{*}{$\begin{array}{l}\text { Sl. } \\
\text { No. }\end{array}$} & \multirow[t]{2}{*}{ Treatments } & \multicolumn{2}{|c|}{ PDI of fruit rot } & \multirow{2}{*}{$\begin{array}{c}\text { PDI of fruit rot } \\
20 \text { days after } \\
2^{\text {nd }} \text { spray }\end{array}$} & \multirow{2}{*}{$\begin{array}{l}\text { Per cent reduction over } \\
\text { control }\end{array}$} \\
\hline & & $\begin{array}{l}\text { Before } 1^{\text {st }} \\
\text { spray }\end{array}$ & $\begin{array}{l}20 \text { days after } 1^{\text {st }} \\
\text { spray }\end{array}$ & & \\
\hline 1 & Mancozeb 75\% WP @ 0.1\% & $\begin{array}{c}50.67 \\
*(45.36)\end{array}$ & $\begin{array}{c}46.67 \\
(43.07)\end{array}$ & $\begin{array}{c}37.33 \\
(37.64)\end{array}$ & 34.88 \\
\hline 2 & Zineb 75\% WP @ 0.1\% & $\begin{array}{c}49.33 \\
(44.60)\end{array}$ & $\begin{array}{c}48.00 \\
(43.83)\end{array}$ & $\begin{array}{c}46.67 \\
(43.07)\end{array}$ & 18.60 \\
\hline 3 & $\begin{array}{c}\text { Carbendazim } 12 \%+\text { mancozeb } 63 \% \\
@ 0.1 \%\end{array}$ & $\begin{array}{c}49.33 \\
(44.60)\end{array}$ & $\begin{array}{c}42.67 \\
(40.76)\end{array}$ & $\begin{array}{c}37.33 \\
(37.64)\end{array}$ & 34.88 \\
\hline 4 & $\begin{array}{c}\text { Tricyclazole } 18 \%+\text { mancozeb } 62 \% @ \\
0.1 \%\end{array}$ & $\begin{array}{c}49.33 \\
(44.60)\end{array}$ & $\begin{array}{c}46.67 \\
(43.07)\end{array}$ & $\begin{array}{c}48.00 \\
(43.83)\end{array}$ & 16.27 \\
\hline 5 & $\begin{array}{c}\text { Carboxin 37.5\% +thiram 37.5\% @ } \\
0.1 \%\end{array}$ & $\begin{array}{c}52.00 \\
(46.13)\end{array}$ & $\begin{array}{c}49.33 \\
(44.60)\end{array}$ & $\begin{array}{c}46.67 \\
(43.07)\end{array}$ & 18.60 \\
\hline 6 & Propiconazole 25\% EC @ 0.025\% & $\begin{array}{c}52.00 \\
(46.13)\end{array}$ & $\begin{array}{c}45.33 \\
(42.30)\end{array}$ & $\begin{array}{c}36.00 \\
(36.84)\end{array}$ & 37.21 \\
\hline 7 & Hexaconazole 5\% EC @ 0.025\% & $\begin{array}{c}52.00 \\
(46.13)\end{array}$ & $\begin{array}{c}49.33 \\
(44.60)\end{array}$ & $\begin{array}{c}45.33 \\
(42.30)\end{array}$ & 20.93 \\
\hline 8 & Iprobenfos 48\% EC @ 0.025\% & $\begin{array}{c}50.67 \\
(45.36)\end{array}$ & $\begin{array}{c}46.67 \\
(43.07)\end{array}$ & $\begin{array}{c}42.67 \\
(40.76)\end{array}$ & 25.58 \\
\hline 9 & Kresoxim methyl 44.3\% EC @ 0.025\% & $\begin{array}{c}48.00 \\
(43.83)\end{array}$ & $\begin{array}{c}45.33 \\
(42.30)\end{array}$ & $\begin{array}{c}42.67 \\
(40.76)\end{array}$ & 25.58 \\
\hline 10 & T.harzianum@1\% & $\begin{array}{c}50.67 \\
(45.36)\end{array}$ & $\begin{array}{c}50.67 \\
(45.36)\end{array}$ & $\begin{array}{c}49.33 \\
(44.60)\end{array}$ & 13.95 \\
\hline 11 & Control & $\begin{array}{c}50.67 \\
(45.36)\end{array}$ & $\begin{array}{c}54.67 \\
(47.66)\end{array}$ & $\begin{array}{c}57.33 \\
(49.20)\end{array}$ & \\
\hline & S.Em \pm & 1.60 & 1.91 & 1.78 & \\
\hline & CD@0.05 & 4.91 & 5.83 & 5.37 & \\
\hline
\end{tabular}

*Arcsine Transformed Values 
Fig.1 In vitro efficacy of contact fungicides against Colletotrichum gloeosporioides

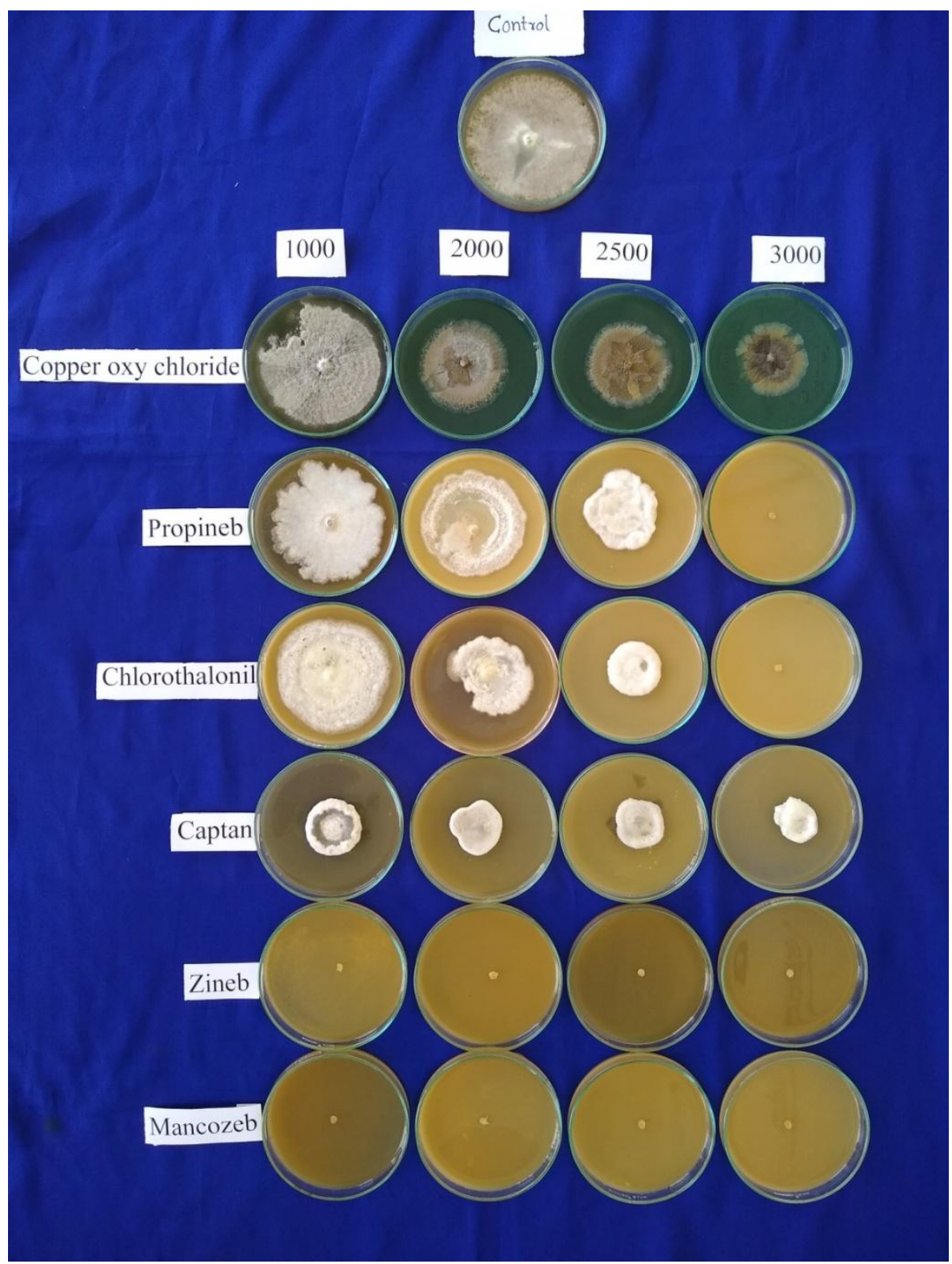


Fig.2 In vitro efficacy of systemic fungicides against Colletotrichum gloeosporioides

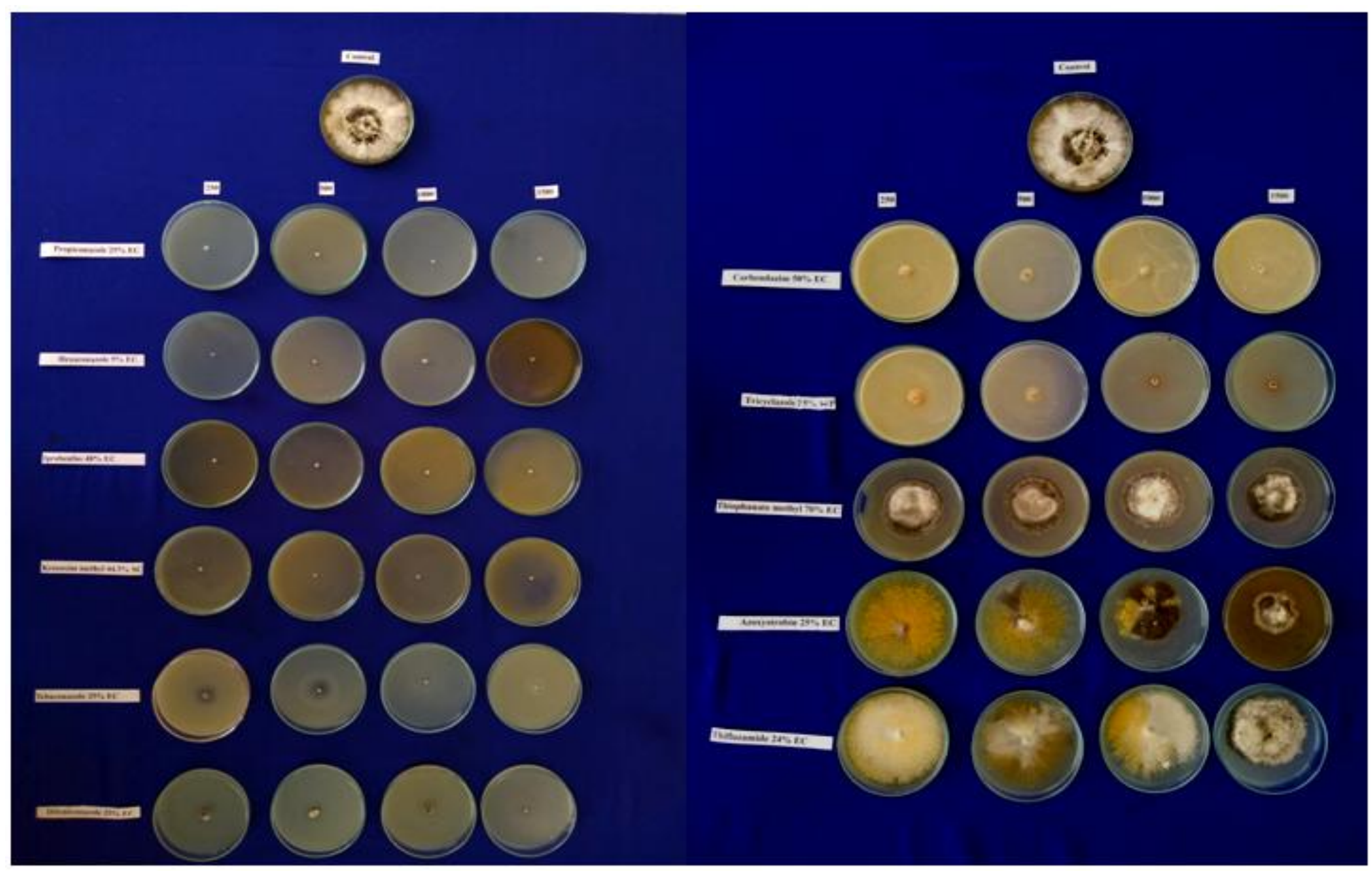


Fig.3 In vitro efficacy of combi products against Colletotrichum gloeosporioides

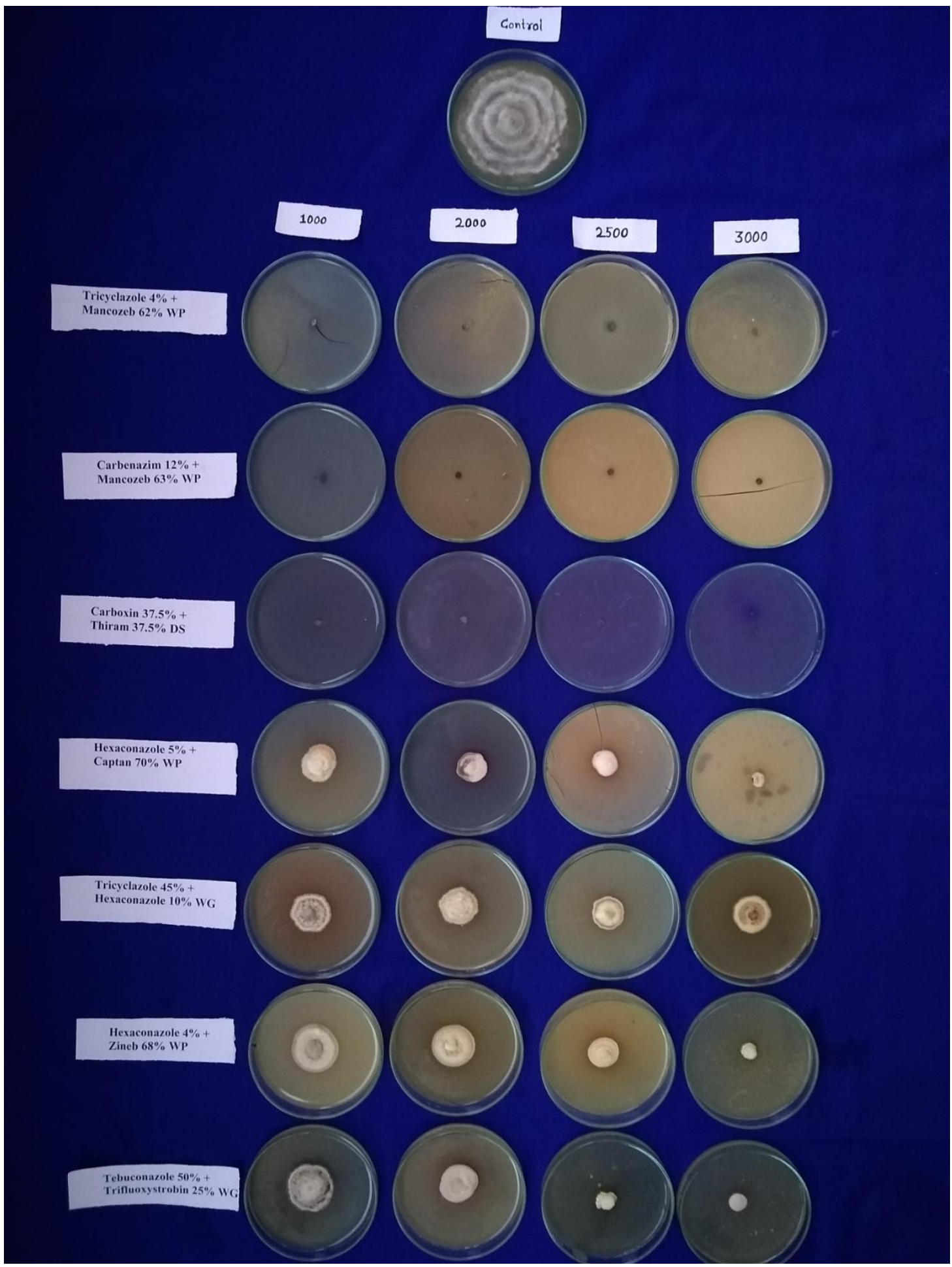


Fig.4 In vitro efficacy of bio agents against Colletotrichum gloeosporioides and Alternaria alternata

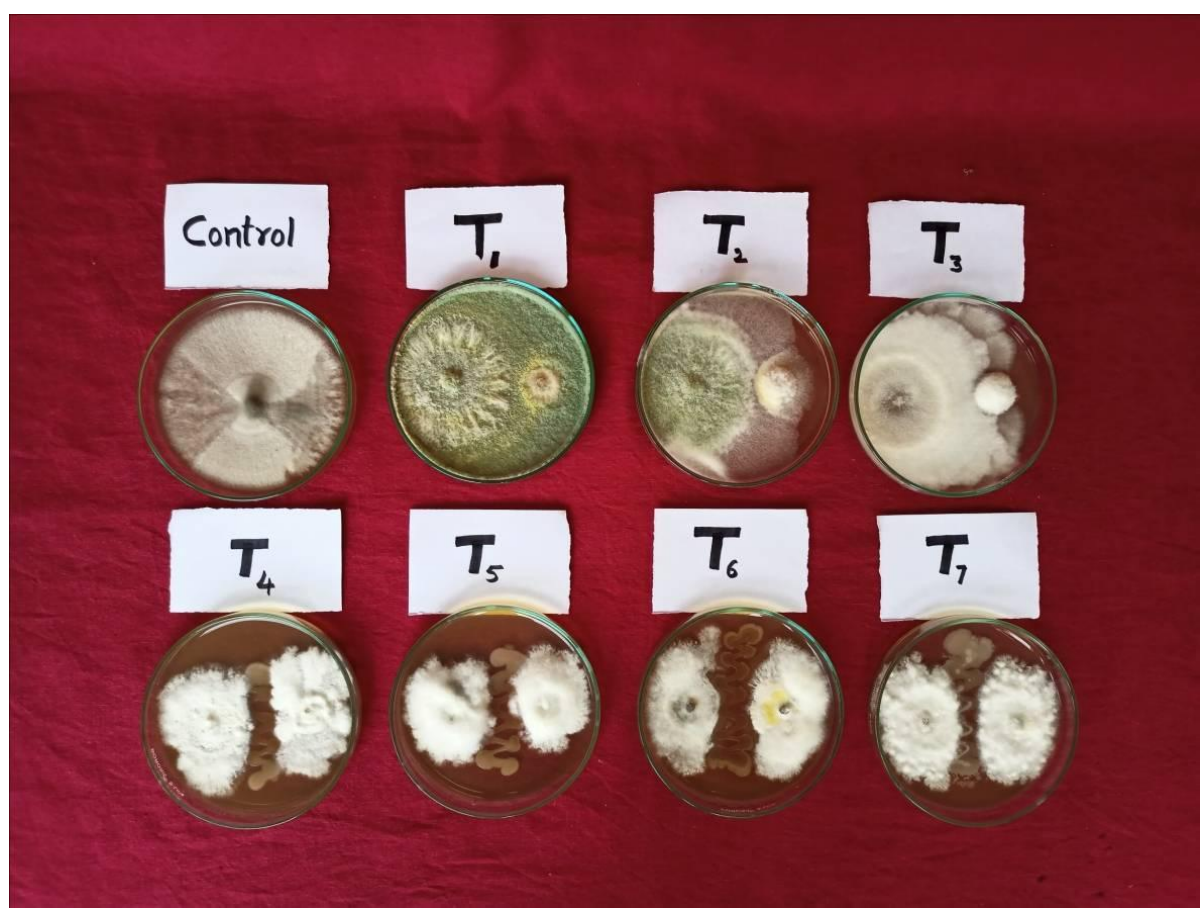

a. Colletotrichum gloeosporioides

T1- T.harzianum (UAS, Dharwad) T2- T.harzianum (UHS Bagalkot)

T3- $T$. viride (UHS, Bagalkot) T4- $P$. fluorescens (UHS, Bagalkot)

T5- $P$. fluorescens (UAS, Dharwad) T6- B. Subtilis (UHS, Baglkot)

T7- B. Subtilis (UAS, Dharwad)

Fig.5 Efficacy of different treatments in management of leaf spot and fruit rot of pomegranate

T1- Mancozeb 75\%WP@0.1\%

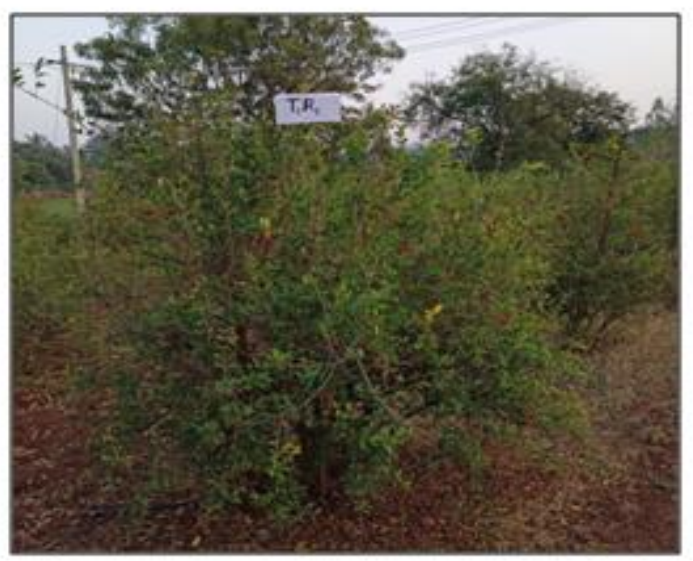

T3- Carbendazim $12 \%+$ mancozeb $63 \% @ 0.1 \%$

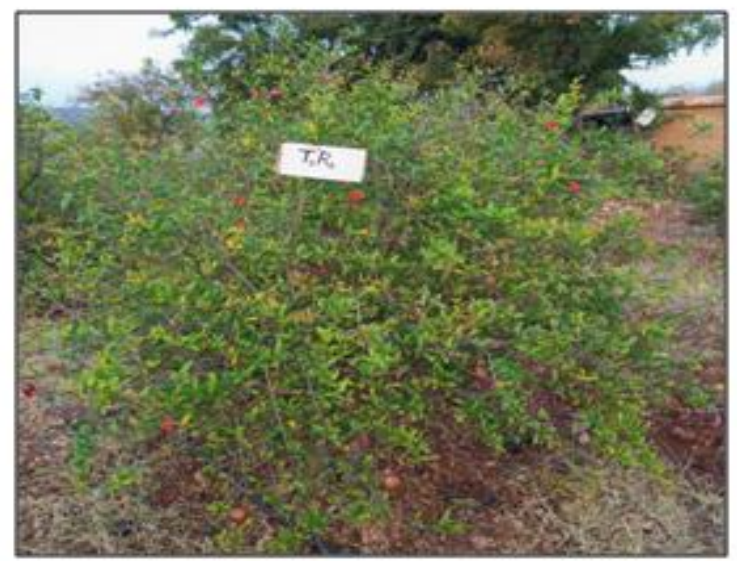


T6- Propiconazole 25\% EC @ 0.025\%

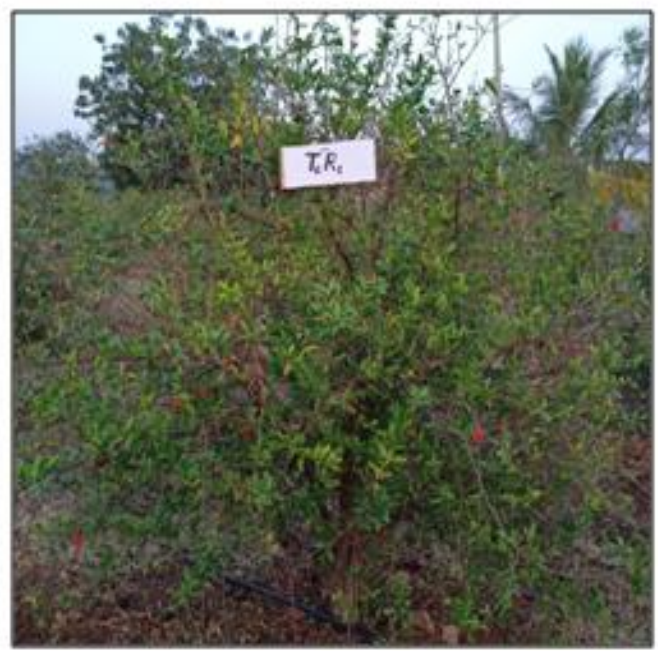

Least inhibition was observed by copper oxy chloride 50\% WP (33.33\%), propineb 70\% WP $(49.62 \%)$ hexaconazole $4 \%$ + zineb $68 \%$ (70.45\%). The results recorded are in line with findings of Jayalkshmi et al., (2013) who reported that carbendizim $12 \%+$ mancozeb $63 \%$ ( 0.3 per cent) and propiconazole $25 \%$ EC $(0.1 \%)$ were most effective in reducing the mycelial growth of the fungus. Naragund $e t$ al., (2012) recorded iprobenfos, propiconazole and carbendazim $12 \%+$ mancozeb $63 \%$ as effective chemicals against Colletotrichum gloeosporioides. Mahesh et al., (2020) reported that SAAF (carbendizim 12\% + mancozeb 63\%) showed $88.40 \%$ inhibition of mycelia growth. Dev and Narendrappa (2016) reported that among systemic fungicides, Hexaconazole 5\% EC, propiconazole 25\% $\mathrm{EC}$, penconazole $10 \% \mathrm{EC}$, tebuconazole $25.9 \%$ EC and carbendazim 50\% EC showed cent per cent mycelial inhibition at 500, 1000 and 2000 ppm concentrations.

Parvathy and Girija (2016) revealed that among seven fungicides maximum inhibition was obtained with tebuconazole $25.9 \%$ EC $(0.10 \%)$ and combination fungicide mancozeb $63 \%+$ carbendazim $12 \%(0.10 \%)$ which gave cent per cent growth inhibition over the

\section{Control}

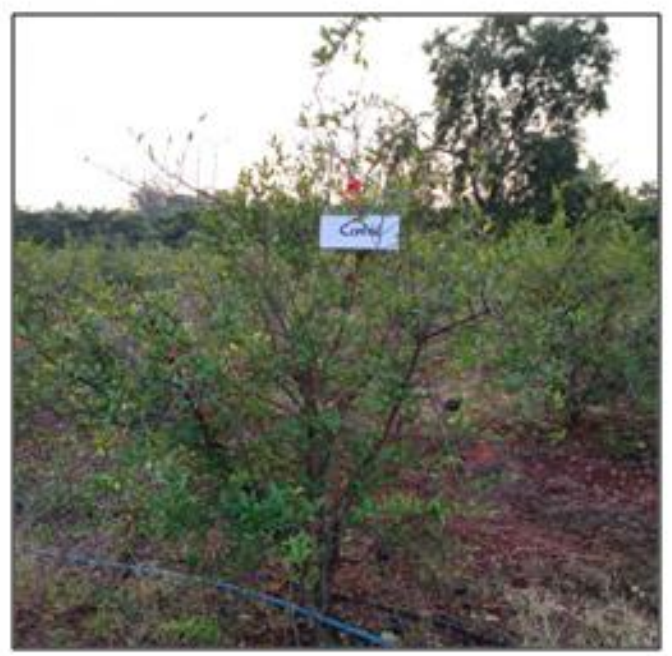

control. Propiconazole 25\% EC, a triazole group fungicide blocks the action of 14- $\alpha-$ sterol demethylase, there by inhibiting the formation of fungal cell membrane ergosterols. Kresoxim methyl $44.3 \%$ EC, which belongs to strobilurin fungicide inhibit the fungal respiration by binding to the cytochrom $b$ complex II and also disrupts the metabolism. Iprobenfos, belongs to organophosphate group interfere with the formation of new cells required for the growth and development of fungus. Mancozeb and zineb are the members of dothiocarbamate fungicide group. These two contact fungicides inactivates the sulfhydryl groups of amino acids and enzymes within fungal cells which results in disruption of respiration and lipid metabolism.

Among different bio agents tested, Trichoderma harzianum, UAS, Dharwad isolate recorded significantly higher per cent mean inhibition (86.67\%) followed by Trichoderma harzianum, UHS, Bagalkot isolate (75.19\%). Trichoderma asperellum, UHS, Bagalkot isolate showed $69.63 \%$ growth inhibition. But in the studies carried out by Sataraddi et al., (2011), Trichoderma asperellum was found to be significant 
followed by Trichoderma harzianum with per cent inhibition of $79.1 \%$ and $62.8 \%$ respectively. The least per cent inhibition was recorded for Bagalkote isolate of Bacillus subtilis $(54.44 \%)$ and is in line with findings of Sataraddi et al., (2011) who recorded least mycelia inhibition by Bacillus subtilis (34.9\%). Naragund et al., (2012) and Dev et al., (2015) reported $T$. asperellum as the effective bio agents against pomegranate anthracnose causing pathogen, Colletotrichum gloeosporioides. Pavitra and Benagi (2017) reported that Tichoderma asperellum and Pseudomonas fluorescens showed maximum fungal inhibition.

The results showed that mancozeb $75 \%$ WP, carbendazim 12\% + mancozeb 63\% and propiconazole $25 \%$ EC @ $0.1 \%$ were effective in controlling the leaf spot and fruit rot incidence as compared to other treatments. $T$. harzianum, UHS, Dharwad showed least per cent disease reduction over control followed by kresoxim methyl 44.3\% EC @ $0.025 \%$. The results are supported by the findings of Jayalakshmi et al., (2015) who reported that carbendazim 12\% + mancozeb 63\%@0.3\% and propiconazole @ $0.1 \%$ reduced the anthracnose disease drastically with high yield. Nargund et al., (2012) reported that propiconazole @ $0.1 \%$ concentration was significantly superior over other fungicides tested in vivo. By this it is clear that two alternate sprays with propiconazole $25 \%$ EC @ $0.025 \%$, mancozeb 75\% WP @ $0.1 \%$ or carbendazim 12\% + mancozeb 63\% @ 0.1\% can reduce fruit rot/ anthracnose incidence in the field.

\section{References}

Anonymous, 2018, Horticultural statistics at a glance 2018, p. 183.

Bhowmik, D., Gopinath, H., Kumar, B. P. and Kumar, K., 2013, Medicinal uses of Punica granatum and its health benefits. J. Pharmacogn. Phytochem., 1(5).

Butler, E., J. 1918, Fungi and Disease in Plants. Thacker Spink and Co. Calcutta, pp. 547.

Chandra S and Tandon R N. 1965, Control of leaf spot of pomegranate with fungicides. Sci. Cult., 31:536.

Dev, D. and Narendrappa, T., 2016, In vitro evaluation of fungicides against Colletotrichum gloeosporioides (Penz.) Penz and Sacc. causing anthracnose of pomegranate (Punica granatum L.). J. Appl. \& Nat. Sci., 8(4): 2268-2272.

Dev, D., Somashekhar Konda, M. E., Tanuja, N., Singh, P. and Narendrappa, T., 2016, In vitro evaluation of bioagents and botanicals against Colletotrichum gloeosporioides (Penz.) Penz \& Sacc. causing anthracnose of Pomegranate. Eco. Env. \& Cons., 22(3): 1229-1232.

Jayalakshmi, K., Nargund, V. B., Raju, J. and Benagi, V. I., 2013, Effect of fungicides and plant extracts on growth of Colletotrichum gloeosporioides (penz.) penz. and Sacc. causing anthracnose of pomegranate. BIOINFOLET, 10(2a): 502-506.

Jayalakshmi, K., Nargund, V. B., Raju, J. and Benagi, V. I., 2015, Effect of fungicides and plant extracts on growth of Colletotrichum gloeosporioides (penz.) penz. and Sacc. causing anthracnose of pomegranate. Bioinfolet, 10(2a): 502506.

Mahesh, M., Venkataravana, P., Reddy, N. G., Devraj, Naik, R. and Priyadarshini, S. K., 2020, In vitro evaluation of different fungicides against Colletotrichum gloeosporioides causing anthracnose of pomegranate. $J$. Entomol. Zool. Stud., 8(4): 642-645.

McRae, W., 1934, Foot rot diseases of Piper 
betle L. In Bengal. Indian J. Agric. Sci., 4: 585-617.

Munhuweyia, K., Lennox, C. L., MeitzHopkins, J. C., Caleb, O. J., Opara, U. L., 2016, Major diseases of pomegranate (Punica granatum L.), their causes and management-A review. Sci. Hortic., 211: 126- 139.

Nargund, V. B., Jayalakshmi, K., Benagi, V. I., Byadgi, A. S., Patil, R. V., Melgarejo, P. and Valero, D., 2012, Status and management of anthracnose of pomegranate in Karnataka State of India. Options Mediterranennes, A,
103: 117-120.

Parvathy, R. and Girija, V. K., 2016, In Vitro evaluation of fungicides and organic preparations against Colletotrichum gloeosporioides causing anthracnose of black pepper (Piper nigrum L.). IJAPSA., 2: 2394-2398.

Sataraddi, A. R., Prashanth, A., Virupaksha Prabhu, H., Jamadar, M. M. and Aski, S., 2011, Role of bio-agents and botanicals in the management of anthracnose of pomegranate. Acta Hort., 890: 539-544.

\section{How to cite this article:}

Vandana, R. K. Mesta, K. C. Kiran Kumar, M. D. Jameel Jhalegar and Siddanna Thoke. 2021. Bio Efficacy of Fungicides and Bio Agents against Colletotrichum gloeosporioides causing Anthracnose in Pomegranate. Int.J.Curr.Microbiol.App.Sci. 10(07): 457-473. doi: https://doi.org/10.20546/ijcmas.2021.1007.051 